\title{
Polycation-sensitive membrane electrode for determination of heparin based on controlled release of protamine
}

\author{
Yan Chen, ${ }^{a b}$ Jiawang Ding ${ }^{a}$ and Wei Qin*a \\ Received 8th February 2012, Accepted 21st February 2012 \\ DOI: 10.1039/c2an35176e
}

\begin{abstract}
A polycation-selective polymeric membrane electrode using dinonylnaphthalene sulfonate as an ion exchanger has been developed as a protamine controlled-release system for potentiometric detection of heparin. The incorporation of tetradodecylammonium tetrakis(4-chlorophenyl)borate as a lipophilic salt in the membrane dramatically improves the sensor's selectivity towards protamine over sodium ions via influencing the activity coefficient of protamine in the membrane, and a stable potential baseline can be obtained in the presence of an electrolyte background. The electrostatic binding interaction between heparin and protamine decreases the concentration of free protamine released at the sample-membrane interface and facilitates the stripping of protamine out of the membrane surface via the ion-exchange process with sodium ions, thus decreasing the membrane potential. Under optimal conditions, the proposed polymeric membrane electrode exhibits a linear relationship between the initial slope of the potential change and the heparin concentration in the range of $0.025-1.25 \mathrm{U} \mathrm{mL}^{-1}$ with an improved detection limit of $0.01 \mathrm{U} \mathrm{mL}^{-1}$.
\end{abstract}

\section{Introduction}

Heparin, a highly-sulfated polysaccharide, is widely used as an injectable anticoagulant during and after surgery. Accurate detection of heparin during such procedures is of great clinical significance. Without close monitoring and control of bloodstream heparin levels, potentially fatal heparin overdose effects, such as internal hemorrhaging, can occur. ${ }^{1}$

Various chemical sensors for heparin detection have been developed using appropriate signal transductions such as spectroscopy, ${ }^{2}$ voltammetry, ${ }^{3}$ quartz microbalance ${ }^{4}$ and conductometry. ${ }^{5}$ Potentiometric sensors are generic and highly successful approaches to chemical sensing, and polyion-selective membrane electrodes have shown to be very promising in biomedical analysis. ${ }^{6-8}$ The development of potentiometric polyion sensors was pioneered by Meyerhoff and coworkers in the 1990s. ${ }^{9-11}$ The polyion potential response of such electrodes is governed by a non-equilibrium steady-state extraction of the polyion into the organic membrane phase via formation of cooperative ion pairs with the lipophilic exchanger in the membrane phase. ${ }^{11,12}$ It has been shown that the developed polyion-sensitive membrane electrodes can be employed to sense heparin as well as protamine even in undiluted human blood. ${ }^{10,13}$

${ }^{a}$ Key Laboratory of Coastal Zone Environmental Processes, Yantai Institute of Coastal Zone Research (YIC), Chinese Academy of Sciences (CAS), Shandong Provincial Key Laboratory of Coastal Zone Environmental Processes, YICCAS, Yantai, Shandong 264003, P. R. China.E-mail: wqin@yic.ac.cn

${ }^{b}$ Graduate University of the Chinese Academy of Sciences, Beijing 100049, P. R. China
By using tridoecylmethylammonium chloride (TDMAC) as the anion exchanger, the polyanion-sensitive membrane electrode exhibits a large and reproducible potentiometric response to heparin. However, lipophilic anions such as thiocyanate and salicylate could strongly interfere with such direct assay of heparin. ${ }^{14}$ Titration of heparin with protamine using the protamine-sensitive membrane electrode as an endpoint detector yields more reliable analytical results than the direct potentiometric assay of heparin with the polyanion sensor, but the procedures to obtain the whole titration curves are rather timeconsuming. ${ }^{13,15,16}$

Recently, our group developed a potentiometric detection system that makes use of outward ion fluxes through an ionselective electrode (ISE) membrane (i.e., fluxes in the direction of the sample solution) to provide controlled-release substrates for in situ biosensing of enzymes and their inhibitors. ${ }^{17,18}$ The potential responses are based on the disturbance of transmembrane diffusion fluxes of the ISEs by the analytes of interest. In the present work, the controlled release of protamine in the polycation-sensitive membrane electrode with dinonylnaphthalene sulfonate (DNNS) as an ion exchanger has been developed for rapid and sensitive determination of heparin. The polycation-sensitive electrode is conditioned with the primary ion (i.e. protamine) to generate an ion flux of protamine from the inner solution to the sample solution under zero-current conditions. It will be shown that the incorporation of tetradodecylammonium tetrakis(4-chlorophenyl)borate (ETH 500) as a lipophilic salt in the polymeric membrane can dramatically improve the sensor's selectivity towards protamine over sodium ions via influencing the activity coefficient of protamine in the 
membrane, thus rendering the potential baseline rather stable in the presence of an electrolyte background. The addition of heparin into the sample solution decreases the concentration of free protamine released at the sample-membrane interface via the strong electrostatic binding interaction between heparin and protamine. This would facilitate the stripping of protamine out of the membrane surface by the ion-exchange process with sodium ions from the sample solution, thus causing a decrease in the measured membrane potential, by which heparin can be sensed potentiometrically (Scheme1).

\section{Experimental section}

\subsection{Materials}

Protamine sulfate (from herring) and unfractionated heparin (from bovine intestinal mucosa) were purchased from Sigma. 2Nitrophenyl octyl ether (o-NPOE), ETH 500, high molecular weight poly(vinyl chloride) (PVC) and DNNS as a 50\% solution in heptane were purchased from Fluka AG (Buchs, Switzerland). Aqueous solutions were prepared with freshly deionized water (18.2 $\mathrm{M} \Omega \mathrm{cm}^{-1}$ specific resistance) obtained with a Pall Cascada laboratory water system.

\subsection{Electrode preparation}

The polycation-sensitive membranes contained $45.5 \mathrm{wt} \% \mathrm{PVC}$, $45.5 \mathrm{wt} \% o$-NPOE, $3.0 \mathrm{wt} \%$ lipophilic cation exchanger DNNS and $6.0 \mathrm{wt} \%$ lipophilic salt ETH 500 (membrane 1). The conventional heparin-selective membranes contained $1.5 \mathrm{wt} \%$ TDMAC, $66 \mathrm{wt} \%$ PVC and $32.5 \mathrm{wt} \%$ DOS (membrane 2). Membranes were obtained by casting a solution of $\sim 200 \mathrm{mg}$ of the membrane components dissolved in $3.0 \mathrm{~mL}$ tetrahydrofuran (THF) into a glass ring of $36 \mathrm{~mm}$ diameter fixed on a glass plate and letting the solvent evaporate overnight. For each ISE, a disk of $7 \mathrm{~mm}$ diameter was punched from the polycation-sensitive membrane and glued to a plasticized PVC tube (i.d. $6 \mathrm{~mm}$, o.d. $9 \mathrm{~mm}$ ) with a THF-PVC slurry. For measurements using the proposed polycation-sensitive electrodes with membrane 1, a $0.05 \mathrm{mg} \mathrm{mL}^{-1}$ protamine solution in $50 \mathrm{mM}$ Tris- $\mathrm{HCl}$ buffer ( $\mathrm{pH}$ 7.4) containing $120 \mathrm{mM} \mathrm{NaCl}$ was added to each electrode as the inner filling solution. Before analysis, the electrodes were conditioned in a solution identical to the inner filling solution for $12 \mathrm{~h}$ at $25 \pm 2{ }^{\circ} \mathrm{C}$. After measurements, the electrodes were regenerated by repeatedly flushing the sensing membranes with
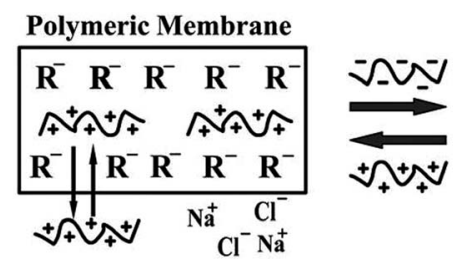

\section{$+4+5$ Protamine 管 Heparin-protamine complex $R^{-}$Ion exchanger}

Scheme 1 Schematic illustration of the potentiometric detection of heparin based on the controlled release of protamine from a protamineconditioned polycation-sensitive membrane electrode. deionized water and soaking the electrodes with a $1.0 \mathrm{mg} \mathrm{mL}^{-1}$ protamine solution in $50 \mathrm{mM}$ Tris- $\mathrm{HCl}$ buffer containing $120 \mathrm{mM} \mathrm{NaCl}$ for $10 \mathrm{~min}$. The electrodes were stored in the conditioning solution at $4{ }^{\circ} \mathrm{C}$ when not in use. For measurements using the conventional heparin-selective electrodes with membrane 2, $50 \mathrm{mM}$ Tris- $\mathrm{HCl}$ buffer containing $120 \mathrm{mM} \mathrm{NaCl}$ was used as the inner filling solution; the electrodes were soaked in the same solution for $12 \mathrm{~h}$ at $25 \pm 2{ }^{\circ} \mathrm{C}$. Unless otherwise specified, all the measurements were done with $50 \mathrm{mM}$ Tris- $\mathrm{HCl}$ buffer of pH 7.4 containing $120 \mathrm{mM} \mathrm{NaCl}$.

\subsection{EMF measurements}

All measurements of electromotive force (EMF) were performed at $25 \pm 2{ }^{\circ} \mathrm{C}$ using a PXSJ-216 pH meter (Shanghai, China) with an $\mathrm{Ag} / \mathrm{AgCl}$ reference electrode in the galvanic cell: $\mathrm{Ag} / \mathrm{AgCl} / 3 \mathrm{M}$ $\mathrm{KCl} /$ sample solution/ISE membrane/inner filling solution/3M $\mathrm{KCl} / \mathrm{AgCl} / \mathrm{Ag}$. To minimize the disturbance of the protamine diffusion through the polymeric membrane by sample stirring, a relatively slow stirring rate of $350 \mathrm{rpm}$ was employed. The membrane electrode was placed $2 \mathrm{~cm}$ above the stir bar. The ISE potential was first measured in $50 \mathrm{mM}$ Tris- $\mathrm{HCl}$ buffer containing $120 \mathrm{mM} \mathrm{NaCl}$ for $5 \mathrm{~min}$ to obtain a stable baseline. After addition of heparin into the buffer solution, the potential change was recorded with time. The initial slope of the potential response was evaluated by a numeric fit of the initial part of the EMF change to a first-order polynomial, and was used for heparin quantification. ${ }^{19}$

\section{Results and discussion}

\subsection{Influence of ETH 500 on the selectivity of the polycation- sensitive membrane electrode}

It has been found that lipophilic salts without ion-exchanger properties such as ETH $500\left(\mathrm{R}^{+} \mathrm{R}^{-}\right)$can be incorporated into solvent polymeric membranes for large improvements in the ISEs' selectivities towards divalent ions. ${ }^{20,21}$ According to DebyeHückel theory, the increase in the membrane ionic strength caused by the addition of lipophilic salts can reduce the activity coefficients for divalent ions stronger than those for monovalent ions, thus enhancing the electrodes's selectivities towards divalent ions against monovalent ions. Similarly, polyion-sensitive membrane electrodes are expected to show a much improved selectivity over monovalent ions upon addition of lipophilic salts, since the reduction in the activity coefficients of polyionic species caused by the increase in ionic strength is much more pronounced than those of monovalent ions. Indeed, this situation is illustrated in Table 1. It can be seen that larger amounts of ETH 500 in the membrane induce higher potential equilibrium responses caused by higher selectivities obtained towards protamine over the interfering ions (i.e. $\mathrm{Na}^{+}$). With addition of $6 \mathrm{wt} \% \mathrm{ETH} \mathrm{500,}$ the polymeric membrane shows an increase of $c a .35 \mathrm{mV}$ in the potential equilibrium response as compared to that of the conventional membrane without ETH 500.

\subsection{Effect of ETH 500 on the potential response to heparin}

The effect of ETH 500 on the heparin response was tested with protamine-conditioned polycation-sensitive membranes which 
Table 1 Potentiometric equilibrium responses for protamine obtained by using DNNS-based PVC membranes doped with various contents of ETH $500^{a}$

\begin{tabular}{ll}
\hline $\begin{array}{l}\text { Amount of ETH } \\
500(\mathrm{wt} \%)\end{array}$ & Equilibrium response $^{b}(\mathrm{mV})$ \\
\hline 0 & $119.2 \pm 1.2$ \\
3 & $145.4 \pm 3.2$ \\
6 & $153.6 \pm 1.1$ \\
10 & $157.5 \pm 1.4$
\end{tabular}

${ }^{a}$ The equilibrium responses were measured with $20 \mu \mathrm{g} \mathrm{mL}^{-1}$ protamine in $120 \mathrm{mM} \mathrm{NaCl}$ background solution until the potentials became constant. The membranes contained $1 \mathrm{wt} \%$ DNNS with PVC and $o$-NPOE (1: 1 by weight). A $120 \mathrm{mM} \mathrm{NaCl}$ solution was added to each electrode as the inner filling medium. Before measurements, the electrodes were conditioned in $120 \mathrm{mM} \mathrm{NaCl}$ overnight at room temperature. ${ }^{b}$ Average value of three determinations \pm standard deviation.

contain different amounts of ETH 500. As shown in Fig. 1A, the membrane without ETH 500 shows a large potential drop of $c a$. $100 \mathrm{mV}$ when exposed to a $120 \mathrm{mM} \mathrm{NaCl}$ background solution, which indicates protamine at the membrane surface can be dramatically stripped out via the ion-exchange process with sodium ions from the sample solution. Indeed, unlike the heparin-sensitive membrane electrode using tridodecylmethylammonium as an anion-exchanger which shows a rather poor reversibility, i.e., inefficient removal of heparin from the phase boundary region of the membrane between measurements by
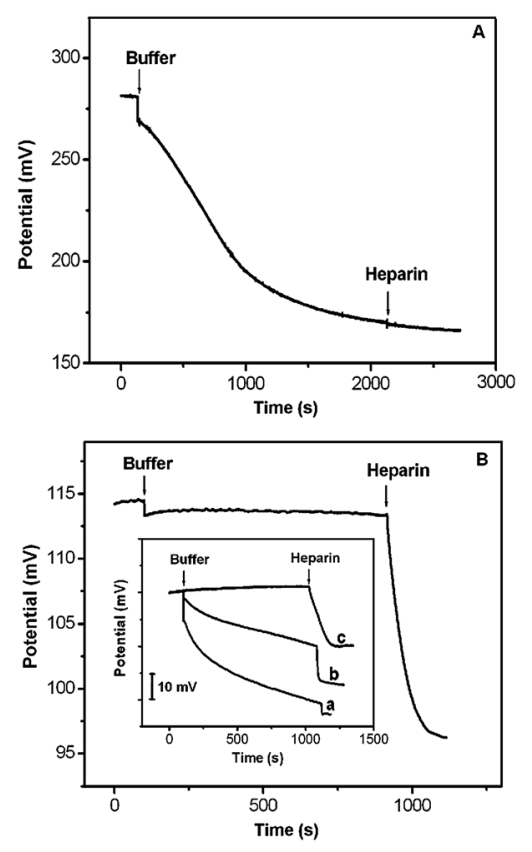

Fig. 1 Effect of the lipophilic salt ETH 500 in the electrode membrane on the potential response to heparin: (A) without ETH 500, and (B) with $6 \mathrm{wt} \%$ ETH 500. The membrane ETH 500 contents in the inset are (a) 1 , (b) 3 , and (c) $10 \mathrm{wt} \%$, respectively. The concentration of heparin added was $0.2 \mathrm{U} \mathrm{mL}^{-1}$. The membranes were composed of $3 \mathrm{wt} \%$ DNNS with PVC and $o$-NPOE $(1: 1$ by weight). The membranes were conditioned in $0.05 \mathrm{mg} \mathrm{mL}^{-1}$ protamine solution in Tris- $\mathrm{HCl}$ buffer containing $120 \mathrm{mM}$ $\mathrm{NaCl}$. placing the electrodes in a high concentration of the background electrolyte, ${ }^{10}$ the enhanced reversibility of the protamine-sensitive membrane electrode is probably due to the small size and hydrophilic nature of protamine relative to heparin. ${ }^{15}$ Fig. 1A also shows that addition of heparin to the sample solution following the potential drop causes a negligible potential change, which implies that most of the cation-exchange sites at the surface of the protamine-conditioned membrane are occupied by sodium ions via the ion-exchange of protamine with the background electrolyte and very little protamine could be stripped out of the membrane surface via the complexation with heparin.

Interestingly, as shown in Fig. 1B, the protamine-conditioned membrane with $6 \mathrm{wt} \%$ ETH 500 exhibits a rather stable potential response when exposed to the electrolyte background, although a small potential drop of $2 \mathrm{mV}$ can be observed shortly after the sample solution is switched. Such behaviors are attributed to the remarkably enhanced selectivity of the ETH 500-incorporated polycation-sensitive membrane for protamine over the interfering ions, which effectively inhibits the ion-exchange process so that sodium ions do not displace a large fraction of the total protamine from the outer boundary layer of the membrane phase. In this case, the membrane potential is mainly determined by the activity of protamine released at the sample-membrane phase boundary via the coextraction process. ${ }^{22}$ Fig. 1B also indicates that the membrane with ETH 500 shows an apparent potential decrease upon addition of heparin into the $120 \mathrm{mM}$ $\mathrm{NaCl}$ background solution. The electrostatic binding interaction between heparin and protamine decreases the concentration of free protamine at the sample-membrane interface and facilitates the stripping of protamine out of the membrane surface via the ion-exchange process with sodium ions, thus decreasing the EMF values. $^{15,23}$

The influence of membrane ETH 500 content on the potential response was shown in the inset of Fig. 1B. It can be seen that the addition of ETH 500 in the membrane renders the baseline of the protamine-conditioned electrode much more stable since the ionexchange of protamine at the membrane surface with sodium ions in the sample solution is effectively inhibited by the increase in membrane ionic strength. Fig. 1B shows that a stable baseline can be obtained when the ETH 500 content increases to $6 \mathrm{wt} \%$, while further increasing the concentration of ETH 500 may cause a lower potential response to heparin. It should be noted that ETH 500, as a kind of ionic liquid, may soften the polycationsensitive membrane if incorporated in larger amounts and would therefore increase the diffusion coefficient of protamine in the polymer membrane phase. Such an effect can promote the ion fluxes of protamine from the inner filling solution into the sample solution and cause higher protamine activities at the samplemembrane interface, which makes the detection of heparin insensitive (see line $\mathrm{c}$ in Fig. 1B for the membrane with $10 \mathrm{wt} \%$ ETH 500).

\subsection{Controlled release of protamine at the sample-membrane interface}

The constant release of protamine under zero-current conditions from the inner filling solution into the sample solution is strongly dependent on the factors influencing either the diffusion coefficient or the concentration profile of protamine in the membrane 
phase. Higher contents of plasticizer promote the diffusion of protamine through the membrane, while higher concentrations of protamine in the inner filling solution induce larger concentration gradients within the membrane. Both of these effects can facilitate the ion fluxes of protamine from the inner solution to the sample solution and therefore increase the protamine concentration at the sample-membrane phase boundary. Since the polycation-sensitive membrane electrode shows the sigmoidal relationship between the measured membrane potential and the protamine concentration in sample solution, ${ }^{13}$ it is essential to modulate the release of protamine so that the potential response to heparin lies in the most sensitive region. As shown in Fig. 2A and Fig. 2B, the membrane with PVC and $o$-NPOE (1:1 by weight) and an inner filling solution of $50 \mu \mathrm{g}$ $\mathrm{mL}^{-1}$ protamine shows a higher potential response to heparin. In this case, the protamine concentration released at the samplemembrane interface can be estimated to be $10 \mu \mathrm{g} \mathrm{mL}^{-1}$, which is based on the potential drop of $2 \mathrm{mV}$ after switching the conditioning solution containing $50 \mu \mathrm{g} \mathrm{mL}^{-1}$ to the $120 \mathrm{mM} \mathrm{NaCl}$ background solution as shown in Fig. $1 \mathrm{~B}$ and on the assumption of an average valence of 20 per protamine molecule with an equilibrium polycation response of $c a .3 \mathrm{mV}$ decade $^{-1}$.

\subsection{Performance of the ETH 500 modified membrane electrode for the determination of heparin}

A typical series of potential response curves showing the dramatic decrease in the initial slope of the EMF change with the increase in heparin concentration are shown in Fig. 3A. Detailed
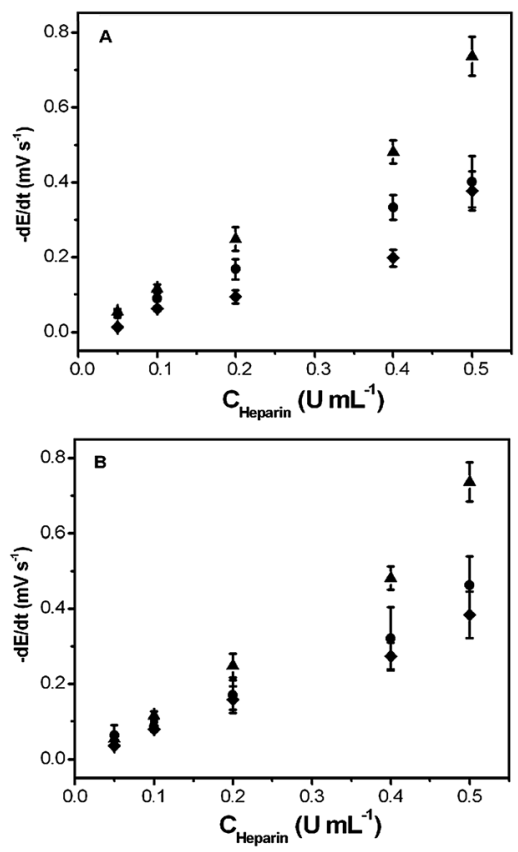

Fig. 2 (A) Potential responses to heparin obtained using protaminesensitive membranes with PVC and $o$-NPOE at different weight ratios: (†) $1: 2,(\mathbf{\Delta}) 1: 1$, and $(\diamond) 2: 1$. (B) Potential responses to heparin obtained using protamine-sensitive membrane electrodes with inner filling solutions of protamine at different concentrations: $(\boldsymbol{O}) 0.01,(\boldsymbol{\Delta})$ 0.05 , and $(\bullet) 0.5 \mathrm{mg} \mathrm{mL}^{-1}$. Error bars represent one standard deviation for three measurements. Other conditions are as in Fig. 1. analysis of the experimental results reveals that there is a linear relationship between the initial slope of the EMF change (dE/dt) and the concentration of added heparin in the range of 0.025 $1.25 \mathrm{U} \mathrm{mL}^{-1}(-\mathrm{dE} / \mathrm{dt}=1.57 \mathrm{C}+0.001, \mathrm{r}=0.9956, \mathrm{dE} / \mathrm{dt}$ in $\mathrm{mV}$ $\mathrm{s}^{-1}, \mathrm{C}$ in $\mathrm{U} \mathrm{mL}^{-1}$ ) (see Fig. 3B). The detection limit of heparin was calculated to be $0.01 \mathrm{U} \mathrm{mL}^{-1}(3 \sigma)$, which is one order of magnitude lower than that of the conventional heparin-sensitive membrane electrode using TDMAC as an ion exchanger. ${ }^{10}$ Although a similar detection limit can be obtained by using the rotating disc membrane electrode, such rotating electrode design suffers from problems of complex instrumentation and large noises in potential signals. ${ }^{24}$ Following each measurement, the membrane electrode can be readily regenerated by conditioning in $1.0 \mathrm{mg} \mathrm{mL} \mathrm{mL}^{-1}$ protamine solution for $10 \mathrm{~min}$. The sensor's reversibility was evaluated by measuring the responses to heparin at a higher $\left(1.0 \mathrm{U} \mathrm{mL}^{-1}\right)$ and lower $\left(0.1 \mathrm{U} \mathrm{mL}^{-1}\right)$ concentrations, respectively. The results are shown in Fig. 4 . It can be seen that the signal changes are fully reversible in both cases. For multiple measurements of $0.4 \mathrm{U} \mathrm{mL}^{-1}$ heparin $(\mathrm{n}=8)$, a relative standard deviation of $6.8 \%$ could be obtained.

\subsection{Interference study}

As illustrated in Fig. 5A, the conventional heparin-selective membrane electrode with membrane 2 shows strong potential responses to lipophilic anions such as thiocyanate $\left(\mathrm{SCN}^{-}\right)$and salicylate $\left(\mathrm{Sal}^{-}\right)$, which would make the direct detection of heparin in blood samples impossible. Although such interferences could be eliminated by titration of heparin with protamine
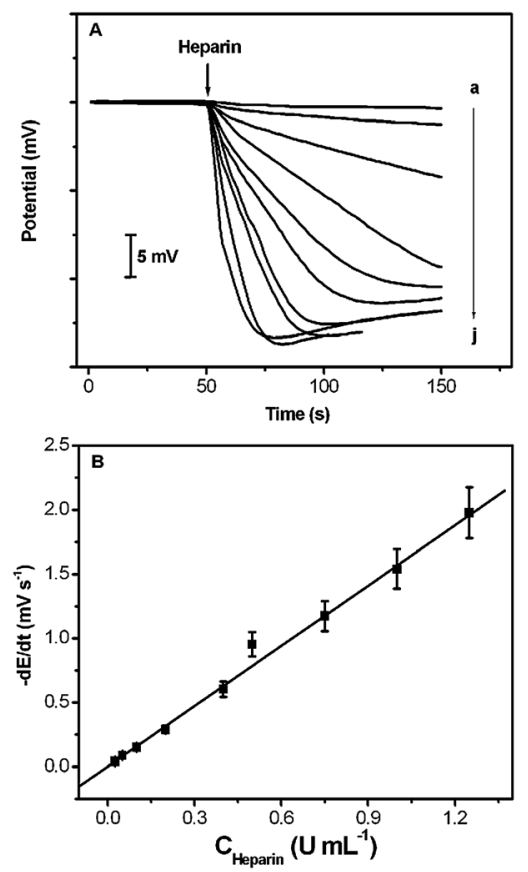

Fig. 3 (A) Typical dynamic potential responses of the proposed polycation-sensitive electrode to various concentrations of heparin: (a) 0.025 , (b) 0.05 , (c) 0.1 , (d) 0.2 , (e) 0.3 , (f) 0.4 , (g) 0.5, (h) 0.75, (i) 1.0, and (j) $1.25 \mathrm{U} \mathrm{mL}^{-1}$. (B) Initial potential response rate as a function of the concentration of heparin. The membrane contained $3 \mathrm{wt} \%$ DNNS, $6 \mathrm{wt} \%$ ETH 500, $45.5 \mathrm{wt} \% \mathrm{PVC}$ and $45.5 \mathrm{wt} \% o$-NPOE. Other conditions are as in Fig. 1. 

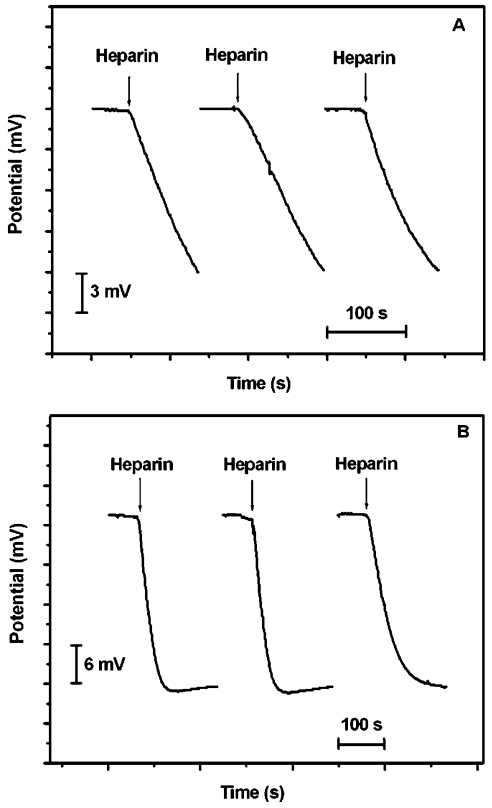

Fig. 4 Recycle potential response profiles for the blank and (A) 0.1 and (B) $1.0 \mathrm{U} \mathrm{mL}^{-1}$ heparin solutions. After each measurement, the polycation-sensitive membrane electrode was regenerated by conditioning in the $1.0 \mathrm{mg} \mathrm{mL}^{-1}$ protamine solution for $10 \mathrm{~min}$. Other conditions are as in Fig. 3.

using the protamine-sensitive membrane electrode as an endpoint detector, the procedures to obtain the whole titration curves are rather time-consuming. Since the proposed potentiometric sensor is based on a polycation-selective membrane

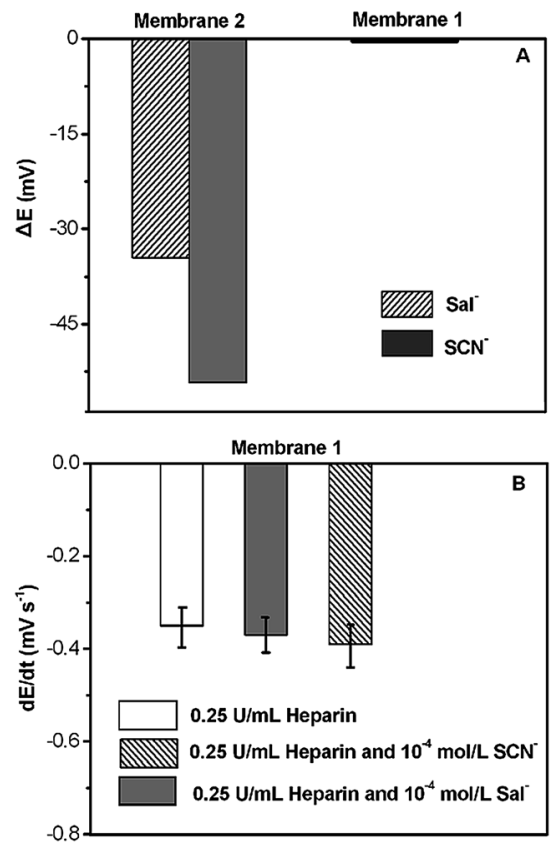

Fig. 5 (A) Potential responses of membranes 1 and 2 to $\mathrm{SCN}^{-}(1 \times$ $\left.10^{-4} \mathrm{M}\right)$ and $\mathrm{Sal}^{-}\left(1 \times 10^{-4} \mathrm{M}\right)$ in $120 \mathrm{mM} \mathrm{NaCl}$ solution. (B) Initial slopes of the EMF responses to $0.25 \mathrm{U} \mathrm{mL}^{-1}$ heparin in the absence and presence of $1 \times 10^{-4} \mathrm{M} \mathrm{SCN}^{-}$or $1 \times 10^{-4} \mathrm{M} \mathrm{Sal}^{-}$in $120 \mathrm{mM} \mathrm{NaCl}$ solution using electrodes with membrane 1 . (membrane 1), $\mathrm{SCN}^{-}$and $\mathrm{Sal}^{-}$cannot be extracted into the membrane due to the presence of the lipophilic cation exchanger in the membrane and no potential responses to these anions were observed (Fig. 5A). In addition, the proposed electrode with controlled release of protamine shows excellent selectivity to heparin in the presence of $\mathrm{SCN}^{-}$or $\mathrm{Sal}^{-}$(Fig. 5B), which is due to the strong binding interaction of protamine with heparin as compared to those with $\mathrm{SCN}^{-}$and $\mathrm{Sal}^{-}$. Such satisfactory selectivity implies that the proposed electrode can be potentially applied to the detection of heparin in biological samples.

\subsection{Application}

To test the feasibility of the proposed sensor, heparin was measured in spiked undiluted pig blood samples containing 0.01 $\mathrm{M}$ citrate. Experimental results showed that there was a linear dependence of the initial slope of the EMF change on the concentration of heparin in the range of $0.4-2 \mathrm{U} \mathrm{mL}^{-1}(-\mathrm{dE} / \mathrm{dt}=$ $0.065 \mathrm{C}-0.017, \mathrm{r}=0.9941, \mathrm{dE} / \mathrm{dt}$ in $\mathrm{mV} \mathrm{s}^{-1}, \mathrm{C}$ in $\mathrm{U} \mathrm{mL}^{-1}$ ) with a detection limit of $0.1 \mathrm{U} \mathrm{mL}^{-1}$ (Fig. 6). For $0.4 \mathrm{U} \mathrm{mL}^{-1}$ heparin, the relative standard deviation was found to be $7.0 \%(n=6)$. The electrode could be used for more than one week without any loss of potential response. It should be noted that lower potential responses of the proposed electrode were observed to viscous blood samples as compared to buffer solution (Fig. 6 vs. Fig. 3). This matrix effect is probably due to the fact that the high
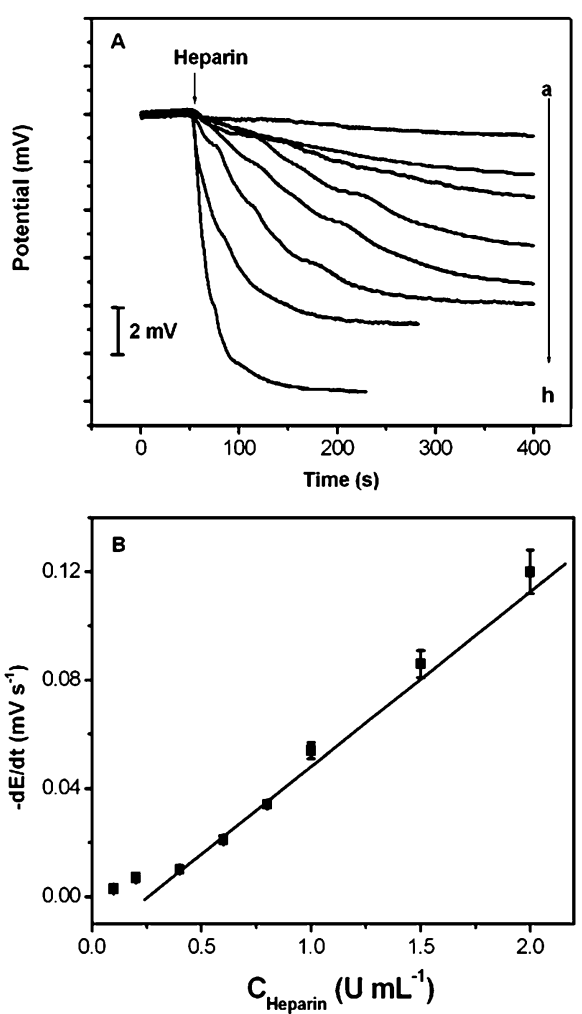

Fig. 6 (A) Typical dynamic potential responses of the proposed polycation-sensitive electrode to heparin spiked in undiluted pig blood samples containing $0.01 \mathrm{M}$ citrate: (a) 0.1 , (b) 0.2 , (c) 0.4 , (d) 0.6 , (e) 0.8 , (f) 1 , (g) 1.5 , (h) $2 \mathrm{U} \mathrm{mL}^{-1}$. (B) Initial potential response rate as a function of the concentration of heparin. Error bars represent one standard deviation for three measurements. Other conditions are as in Fig. 3. 
viscosity of blood can decrease the diffusion coefficient of the polyion and lower the mass transport of protamine from the membrane to the sample solution. ${ }^{16}$

\section{Conclusions}

A novel polycation-selective polymeric membrane electrode has been developed as a protamine controlled-release system for potentiometric detection of heparin. The proposed membrane electrode offers several advantages over other polyion sensors for heparin determination. Firstly, the present sensor exhibits high sensitivity with a detection limit being one order of magnitude lower than that of the conventional heparin-sensitive membrane electrode. Secondly, as compared to the tedious procedures for the potentiometric titration of heparin with protamine using the protamine-sensitive membrane electrode as the endpoint detector, a more rapid analysis can be obtained with the proposed method since only the initial response slope of the potential change (within $60 \mathrm{~s}$ upon addition of heparin) needs to be measured for heparin quantification. Thirdly, in contrast to the conventional polyion sensor configuration for which the protamine-sensitive polymeric membrane electrode is conditioned with the highly discriminated ion (i.e. $\mathrm{Na}^{+}$), the present protamine-sensitive electrode is conditioned with the primary ion (i.e. protamine) to generate an ion flux of protamine from the inner solution to the sample solution under zero-current conditions. Such a protamine controlled-release system does not require manual addition of protamine to the sample solution, thus making the operation very convenient. Further efforts to evaluate the clinical utility of this new sensing platform for the determination of unfractionated or low molecular weight heparins in human blood samples are currently in progress in our laboratory.

\section{Acknowledgements}

This work was financially supported by the National Natural Science Foundation of China (No. 41176081), the Outstanding Youth Natural Science Foundation of Shandong Province (No.
JQ200814), and the Taishan Scholar Program of Shandong Province (No. TS20081159).

\section{References}

1 S. T. Olson, I. Bjork, R. Sheffer, P. A. Craig, J. D. Shore and J. Choay, J. Biol. Chem., 1992, 267, 12528-12538.

2 R. Y. Zhan, Z. Fang and B. Liu, Anal. Chem., 2010, 82, 1326-1333.

3 J. D. Guo and S. Amemiya, Anal. Chem., 2006, 78, 6893-6902.

4 T. J. Cheng, T. M. Lin and H. C. Chang, Anal. Chim. Acta, 2002, 462, 261-273.

5 A. Trojanek, J. Langmaier, E. Samcova and Z. Samec, J. Electroanal. Chem., 2007, 603, 235-242.

6 L. Wang, S. Buchanan and M. E. Meyerhoff, Anal. Chem., 2008, 80, 9845-9847.

7 L. Wang and M. E. Meyerhoff, Electroanal., 2010, 22, 26-30.

8 Y. Kang, K. Gwon, J. H. Shin, H. Nam, M. E. Meyerhoff and G. S. Cha, Anal. Chem., 2011, 83, 3957-3962.

9 S. C. Ma, V. C. Yang and M. E. Meyerhoff, Anal. Chem., 1992, 64, 694-697.

10 S. C. Ma, V. C. Yang, B. Fu and M. E. Meyerhoff, Anal. Chem., 1993, 65, 2078-2084.

11 B. Fu, E. Bakker, V. C. Yang and M. E. Meyerhoff, Macromolecules, 1995, 28, 5834-5840.

12 B. Fu, E. Bakker, J. H. Yun, V. C. Yang and M. E. Meyerhoff, Anal. Chem., 1994, 66, 2250-2259.

13 N. Ramamurthy, N. Baliga, J. A. Wahr, U. Schaller, V. C. Yang and M. E. Meyerhoff, Clin. Chem., 1998, 44, 606-613.

14 K. L. Gemene and M. E. Meyerhoff, Anal. Chem., 2010, 82, 16121615.

15 J. H. Yun, M. E. Meyerhoff and V. C. Yang, Anal. Biochem., 1995, 224, 212-220.

16 W. Qin, W. Zhang, K. P. Xiao and M. E. Meyerhoff, Anal. Bioanal. Chem., 2003, 377, 929-936.

17 J. W. Ding and W. Qin, J. Am. Chem. Soc., 2009, 131, 14640-14641.

18 J. W. Ding and W. Qin, Chem. Commun., 2009, 971-973.

19 J. Langmaier, E. Samcová and Z. Samec, Anal. Chem., 2007, 79, $2892-2900$.

20 M. Nägele, Y. M. Mi, E. Bakker and E. Pretsch, Anal. Chem., 1998, 70, 1686-1691.

21 B. K. Oh, C. Y. Kim, H. J. Lee, K. L. Rho, G. S. Cha and H. Nam, Anal. Chem., 1996, 68, 503-508.

22 T. Sokalski, T. Zwickl, E. Bakker and E. Pretsch, Anal. Chem., 1999, 71, 1204-1209.

23 J. W. Ding, Y. Chen, X. W. Wang and W. Qin, Anal. Chem., 2012, 84, 2055.

24 Q. S. Ye and M. E. Meyerhoff, Anal. Chem., 2001, 73, 332-336. 